\title{
The macula densa is worth its salt
}

National Institute of Diabetes, Digestive, and Kidney Diseases, National Institutes of Health, Bethesda, Maryland 20892, USA

Address correspondence to: Jürgen Schnermann, National Institute of Diabetes, Digestive, and Kidney Diseases, National Institutes of Health, Building 10, Room 4D51, 10 Center Drive MSC 1370, Bethesda, Maryland 20892-1370, USA.

Phone: (301) 435-6580; Fax: (301) 435-6587; E-mail: JurgenS@intra.niddk.nih.gov.

Ultrafiltration of plasma in the glomerulus and the subsequent absorption of water and solutes across the tubular epithelium have evolved as the fundamental processes responsible for formation of urine by the vertebrate kidney. In mammals, this filtration/absorption process is remarkable for its magnitude. The entire extracellular fluid volume is filtered through the renal glomeruli 15 times per day in humans, 50 times per day in rats, and 100 times per day in mice. Almost all the filtered salt and water is absorbed by the renal tubules, so that the volume of excreted urine is usually less than one hundredth of the filtrate volume.

Whatever the evolutionary advantages of this design, it is evident that it carries inherent vulnerability: salt excretion and, therefore, maintenance of extracellular fluid volume, circulatory volume, and organ perfusion become critically dependent upon a perfect match between the rate of ultrafiltration and tubular absorption. Catastrophic salt losses and salt gains by the kidneys are prevented by a set of mechanistic interactions between filtration and absorption. Changes in filtration lead to changes in absorption, and changes in tubular transport cause changes in filtration, relationships described as glomerular-tubular balance and tubuloglomerular feedback (TGF), respectively $(1,2)$. Protective adjustments in the filtration/absorption match are so much a part of reg- ulation of kidney function that massive salt losses through the kidneys the renal equivalent of cholera rarely occur.

Just how effective these adjustments are has been recently underscored by the demonstration that genetic defects in major renal salt and fluid transporters have surprisingly small consequences for body $\mathrm{Na}$ balance. Even though transporters such as the $\mathrm{Na}-\mathrm{K}-2 \mathrm{Cl}$ cotransporter, the $\mathrm{NaCl}$ cotransporter, the $\mathrm{ClC}-\mathrm{Kb} \mathrm{Cl}$ channel, aquaporin 1 , and $\mathrm{NHE}_{3}$ are each responsible for the absorption of major fractions of filtered salt and water, their complete absence is fully compatible with maintenance of $\mathrm{Na}$ balance, albeit at a somewhat reduced level of extracellular fluid volume (3-7). The compensatory mechanisms that prevent progressive and fatal volume depletion in these states of renal $\mathrm{NaCl}$ and water malabsorption are remarkably similar to those activated by extreme dietary Na restriction - for example, in the "no-salt" culture of the Yanomamo people (8).

The macula densa and the TGF response. TGF, a major mechanism by which gross filtration/absorption mismatches are prevented, is the subject of the article by Thomson et al. in this issue of the JCI (9). As shown schematically in Figure 1, in every mammalian nephron, the distal tubule is attached to its glomerulus of origin at a point where normally about $80 \%$ of the filtered water and $85 \%$ of the filtered $\mathrm{Na}$ has been reabsorbed. The epithelial cells at this contact point, the macula densa cells, together with the smooth muscle cells of the afferent arterioles and the reninsecreting granular cells, form a functional unit, the juxtaglomerular apparatus (JGA). Functionally, the macula densa serves as a sensor of luminal $\mathrm{NaCl}$. Concentrationdependent salt uptake through the $\mathrm{Na}-\mathrm{K}-2 \mathrm{Cl}$ cotransporter in the apical membrane of macula densa cells initiates a sequence of events that regulates afferent arteriolar tone and thereby filtration rate (10). As pointed out later, the $\mathrm{NaCl}$ signal also controls the rate of renin secretion from juxtaglomerular granular cells (11). With increases in luminal $\mathrm{NaCl}$ concentration, glomerular fil-

\section{Figure 1}

Schematic drawing of the architecture of a nephron, showing the position of the macula densa in the nephron and its relationship to the glomerulus of origin. A magnified view of the contact area of the macula densa with the glomerulus is shown on the right. MD, macula densa; AA, afferent arteriole; EA, efferent arteriole, GC: granular cell, SMC: smooth muscle cell.






\section{Figure 2}

Relationship between glomerular filtration rate and the tubular flow rate at the end of the proximal tubule. Late proximal flow rate is used as an experimentally controllable surrogate for the true TGF signal, the $\mathrm{NaCl}$ concentration at the macula densa. Values given are valid for the rat. Arrows with open heads indicate increasing levels of nitric oxide and angiotensin II. The solid line represents the relationship for the control state; the dotted lines indicate resetting of the TGF curve to the right following prolonged administration of benzolamide or ECV expansion or to the left following ECV depletion. The open circles indicate the operating points for the different conditions. ECV, extracellular volume.

tration rate (GFR) decreases in an inverse and highly nonlinear fashion (Figure 2). The operating point for this response is located in the steep part of the feedback curve, so that an elevation of salt concentration results in vasoconstriction and a reduction in GFR, whereas decreased salt concentration causes vasodilatation and an increase in GFR $(12,13)$. This homeostatic feedback loop minimizes variations of salt concentration in the fluid entering the capacity-limited distal portions of the nephron. It thereby prevents filtration rate-dependent changes in urinary $\mathrm{Na}$ excretion that could result in dysregulation of the extracellular fluid volume.

Long-term modulation of the TGF response. A fixed relationship between GFR and distal salt concentration would be maladaptive, when $\mathrm{NaCl}$ concentration changes at the macula densa do not arise from an acute perturbation but from a long-standing primary disturbance in body fluid volume. Expansion of the extracellular fluid volume reduces proximal salt absorption and elevates macula densa $\mathrm{NaCl}$ concentration, and it would therefore decrease GFR by TGF activation. Conversely, volume depletion would be predicted to increase GFR. Neither a decrease in GFR with volume expansion nor an increase with volume depletion would be advantageous, and neither in fact takes place. Evidently, the TGF curve is adjusted, shifting the operating point to the right in volume expansion or to the left in volume depletion (1). The necessity for the TGF mechanism to have a flexible set point has been recognized for some time (14).

The results by Thomson et al. in this issue of the JCI, together with previous work by the same group,

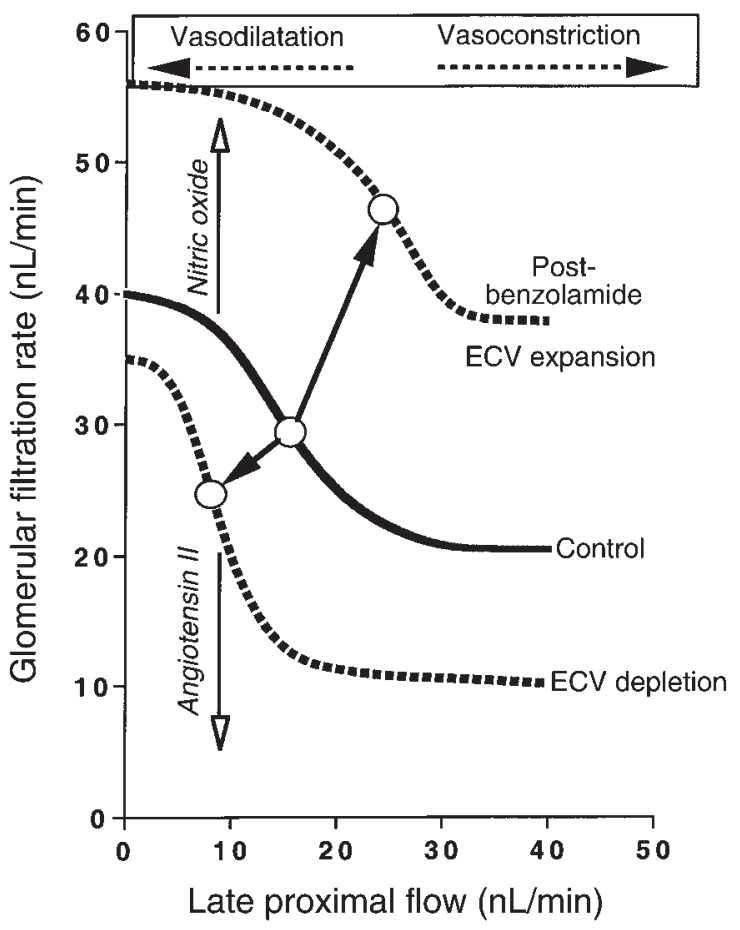

provide insights into the mechanisms responsible for TGF resetting $(9,15)$. These authors have shown earlier that an acute inhibition of proximal $\mathrm{NaHCO}_{3}$ absorption by the carbonic anhydrase inhibitor benzolamide causes an increase in salt delivery to the macula densa, accompanied by the predicted TGF-mediated reduction in filtration rate (16). Their new results demonstrate that repeated administration of benzolamide over 24 hours, which results in prolonged hyperperfusion of the macula densa, induces a rightward and upward shift of the TGF curve (Figure 2). As a consequence of this shift, GFR in the post-benzolamide phase was actually higher than in control. The hyperfiltration and the pattern of TGF resetting caused by benzolamide are strikingly similar to those seen previously during acute extracellular volume expansion, another state in which the macula densa is exposed for some time to an increase in $\mathrm{NaCl}$ concentration (13, 17). Interestingly, the benzolamideinduced TGF resetting outlasted the actual duration of the high perfusion stimulus. As the authors point out, it is easy to comprehend that such a memory effect would facilitate the excretion of $\mathrm{NaCl}$ and shorten the time required to correct any primary increase in the $\mathrm{Na}$ content of the body. It is more difficult to see how persistent hyperfiltration and TGF resetting could be advantageous in the situation of the current study, where relative volume depletion must have been caused by the repeated injection of the carbonic anhydrase inhibitor. Thus, a history of hyperperfusion of the macula densa appears to cause hyperfiltration regardless of the state of the extracellular volume, indicating that TGF resetting in the post-benzolamide phase is determined by local, not systemic, factors.

Regulators of the TGF set point. Resetting of the TGF function curve occurs in a number of different conditions associated with prolonged hyper- or hypoperfusion of the macula densa. The renin-angiotensin system (RAS) is one of the mechanisms that have been shown to alter TGF curve characteristics: increased angiotensin II levels are associated with a left shift, and decreased angiotensin II with a right shift, of the feedback curve (18). This pattern of TGF adaptation by angiotensin II is compatible with the notion that changes in the activity of the RAS contribute to TGF resetting during changes in extracellular fluid volume. Because exposure of macula densa cells to altered $\mathrm{NaCl}$ regulates renin secretion in an appropriate manner (11), it is attractive to view this response as the mechanism that 
couples macula densa $\mathrm{NaCl}$ to the resetting of the TGF.

Whereas the vasoconstrictor angiotensin II enhances TGF responsiveness, the release of the vasodilator nitric oxide (NO) into the JGA tonically attenuates TGF-induced vasoconstriction (19). Macula densa cells express high levels of NOS-I, the neuronal isoform of nitric oxide synthase, and are a likely source of NO acting in the JGA $(19,20)$. The paper by Thomson et al. shows that NO contributes to the persistent resetting following benzolamide administration, since hyperfiltration is prevented by systemic or intratubular application of the NOS-I inhibitors 7-nitroindazole and S-methyl-thiocitrulline. The increase in NOS-I protein expression in macula densa cells furnishes a possible mechanism for upregulation of $\mathrm{NO}$ production in the post-benzolamide phase. Overall, the experiments performed by Thomson et al. clearly demonstrate that, after prolonged benzolamideinduced hyperperfusion of the macula densa, NO-mediated vasodilatation shifts the TGF curve to the right without interfering with the ability of the TGF mechanism to regulate GFR around the new operating point. To what extent NO contributes to TGF resetting under other conditions remains an open question. It is of note that in two chronic models of proximal tubule transport inhibition, in $A Q P 1$ and $\mathrm{NHE}_{3}$ knockout mice, the TGF mechanism causes a reduction in filtration rate, suggesting that in these cases of ongoing proximal malabsorption, TGF does not appear to reset to the right and upward $(3,7)$.
In summary, the study by Thomson and coworkers in this issue contributes to our understanding of the complexity of GFR regulation by the macula densa and the JGA. The macula densa acts as a sensor of $\mathrm{NaCl}$ concentration in TGF, the mechanism critical for the rapid adaptation of filtration rate to the absorptive capacity of the tubule. When prolonged deviations in $\mathrm{NaCl}$ concentration signal a state of salt deficit or excess, the macula densa resets the operating point of the TGF loop by altering the local balance of vasoconstrictors and vasodilators, particularly angiotensin II and NO. Salt at the macula densa is the ingredient which permits the kidney to enjoy the benefits of a very high filtration rate without an undue risk of inappropriate salt loss or salt retention.

1. Briggs, J.P., and Schnermann, J. 1995. Contro of renin release and glomerular vascular tone by the juxtaglomerular apparatus. In Hypertension. Volume 1. J.H. Laragh and B.M. Brenner, editors. Raven Press. New York, NY 1359-1383.

2. Wilcox, C., Baylis, C., and Wingo, C. 1992 Glomerular-tubular balance and proximal regulation. In The kidney: physiology and pathophysiology. Volume 2. DW Seldin and G. Giebisch, editors. Raven Press. New York, NY 1807-1841.

3. Lorenz, J.N., Schultheis, P.J., Traynor, T., Shull, G.E., and Schnermann, J. 1999. Micropuncture analysis of single-nephron function in NHE3-deficient mice. Am. J. Physiol. 277:F447-F453.

4. Simon, D.B., et al. 1996. Bartter's syndrome, hypokalaemic alkalosis with hypercalciuria, is caused by mutations in the $\mathrm{Na}-\mathrm{K}-2 \mathrm{Cl}$ cotransporter NKCC2. Nat. Genet. 13:183-188.

5. Simon, D.B., et al. 1996. Gitelman's variant of Bartter's syndrome, inherited hypokalaemic alkalosis, is caused by mutations in the thiazide-sensitive $\mathrm{Na}-\mathrm{Cl}$ cotransporter. Nat. Genet. 12:24-30.

6. Simon, D.B., et al. 1997. Mutations in the chloride channel gene, CLCNKB, cause Bartter's syndrome type III. Nat. Genet
17:171-178.

7. Schnermann, J., et al. 1998. Defective proximal tubular fluid reabsorption in transgenic aquaporin-1 null mice. Proc. Natl. Acad. Sci. USA. 95:9660-9664.

8. Oliver, W.J., Neel, J.V., Grekin, R.J., and Cohen, E.L. 1981. Hormonal adaptation to the stresses imposed upon sodium balance by pregnancy and lactation in the Yanomama Indians, a culture without salt. Circulation. 63:110-116.

9. Thomson, S.C., et al. 1999. Temporal adjustment of the juxtaglomerular apparatus during sustained inhibition of proximal reabsorption. J. Clin. Invest. 104:1149-1158.

10. Wright, F.S., and Schnermann, J. 1974. Interference with feedback control of glomerular filtration rate by furosemide, triflocin, and cyanide. J. Clin. Invest. 53:1695-1708.

11. Skott, O., and Briggs, J.P. 1987. Direct demonstration of macula densa-mediated renin secretion. Science. 237:1618-1620.

12. Briggs, J.P., Schubert, G., and Schnermann, J. 1984. Quantitative characterization of the tubuloglomerular feedback response: effect of growth. Am. J. Physiol. 247:F808-F815.

13. Thomson, S.C., and Blantz, R.C. 1993. Homeostatic efficiency of tubuloglomerular feedback in hydropenia, euvolemia, and acute volume expansion. Am. J. Physiol. 264:F930-F936.

14. Thurau, K., and Schnermann, J. 1998. The Na concentration at the macula densa cells as a factor regulating glomerular filtration rate (micropuncture studies). 1965 [reprint]. J. Am. Soc. Nephrol. 9:925-934.

15. Thomson, S.C., Vallon, V., and Blantz, R.C. 1997. Reduced proximal reabsorption resets tubuloglomerular feedback in euvolemic rats. Am. J. Physiol. 273:R1414-R1420.

16. Tucker, B.J., Steiner, R.W., Gushwa, L.C., and Blantz, R.C. 1978. Studies on the tubuloglomerular feedback system in the rat. The mechanism of reduction in filtration rate with benzolamide. J. Clin. Invest. 62:993-1004.

17. Davis, J.M., et al. 1988. Increased tubuloglomerular feed-back mediated suppression of glomerular filtration during acute volume expansion in rats. J. Physiol. (Lond.) 395:553-576.

18. Schnermann, J. 1998. Juxtaglomerular cell complex in the regulation of renal salt excretion. Am. J. Physiol. 274:R263-R279.

19. Wilcox, C.S., et al. 1992. Nitric oxide synthase in macula densa regulates glomerular capillary pressure. Proc. Natl. Acad. Sci. USA. 89:11993-11997.

20. Mundel, P., et al. 1992. Expression of nitric oxide synthase in kidney macula densa cells. Kidney Int. 42:1017-1019. 